\title{
A QM-MD simulation approach to the analysis of FRET processes in (bio)molecular systems. A case study: complexes of $E$. coli purine nucleoside phosphorylase and its mutants with formycin $A$
}

\author{
M. Sobieraj • K. A. Krzyśko • A. Jarmula • \\ M. W. Kalinowski • B. Lesyng • M. Prokopowicz • \\ J. Cieśla • A. Gojdź • B. Kierdaszuk
}

Received: 4 November 2014 / Accepted: 1 February 2015 / Published online: 10 March 2015

(C) The Author(s) 2015. This article is published with open access at Springerlink.com

\begin{abstract}
Predicting FRET pathways in proteins using computer simulation techniques is very important for reliable interpretation of experimental data. A novel and relatively simple methodology has been developed and applied to purine nucleoside phosphorylase (PNP) complexed with a fluorescent ligand - formycin A (FA). FRET occurs between an excited Tyr residue (D*) and FA (A). This study aims to interpret experimental data that, among others, suggests the absence of FRET for the PNPF159A mutant in complex with FA, based on novel theoretical methodology. MD simulations for the protein molecule containing $\mathrm{D}^{*}$, and complexed with $\mathrm{A}$, are carried out. Interactions of $\mathrm{D}^{*}$ with its molecular environment are accounted by including changes of the ESP
\end{abstract}

Co-authors M. Sobieraj and M. Prokopowicz equally contributed to this work. They are first co-authors from the theoretical (M. Sobieraj, K. A. Krzyśko, A. Jarmuła, M. W. Kalinowski, B. Lesyng) and experimental (M. Prokopowicz, J. Cieśla, A. Gojdź, B. Kierdaszuk) groups, respectively.

This paper belongs to Topical Collection 6th conference on Modeling \& Design of Molecular Materials in Kudowa Zdrój (MDMM 2014)

M. Sobieraj · K. A. Krzyśko • B. Lesyng $(\bowtie) \cdot$ M. Prokopowicz B. Kierdaszuk $(\bowtie)$

Department of Biophysics, Institute of Experimental Physics, Faculty of Physics, University of Warsaw, 02-089 Warsaw, Poland e-mail: lesyng@gmail.com

e-mail: borys@biogeo.uw.edu.pl

K. A. Krzyśko • M. W. Kalinowski • B. Lesyng Bioinformatics Laboratory, Medical Research Centre,

Polish Academy of Sciences, 02-106 Warsaw, Poland

A. Jarmuła

Department of Biochemistry, Nencki Institute of Experimental

Biology, Polish Academy of Sciences, 02-093 Warsaw, Poland

J. Cieśla · A. Gojdź

Department of Technology and Biotechnology, Faculty of Chemistry,

Warsaw University of Technology, 00-664 Warsaw, Poland charges in $\mathrm{S}_{1}$, compared to $\mathrm{S}_{0}$, and computed at the SCF-CI level. FRET probability $W_{F}$ depends on the inverse six-power of the D*-A distance, $R_{d a}$. The orientational factor $0<\mathrm{k}^{2}<4$ between $\mathrm{D}^{*}$ and $\mathrm{A}$ is computed and included in the analysis. Finally $W_{F}$ is time-averaged over the MD trajectories resulting in its mean value. The red-shift of the tyrosinate anion emission and thus lack of spectral overlap integral and thermal energy dissipation are the reasons for the FRET absence in the studied mutants at $\mathrm{pH} 7$ and above. The presence of the tyrosinate anion results in a competitive energy dissipation channel and red-shifted emission, thus in consequence in the absence of FRET. These studies also indicate an important role of the phenyl ring of Phe159 for FRET in the wild-type PNP, which does not exist in the Ala159 mutant, and for the effective association of PNP with FA. In a more general context, our observations point out very interesting and biologically important properties of the tyrosine residue in its excited state, which may undergo spontaneous deprotonation in the biomolecular systems, resulting further in unexpected physical and/or biological phenomena. Until now, this observation has not been widely discussed in the literature.

Keywords Deprotonation · Emission spectroscopy · Excited state · Formycin A · FRET · PNP · QC-MD · SCF-CI $\cdot$ Simulations $\cdot$ Tyrosinate anion

$\begin{array}{ll}\text { Abbreviations } & \\ \text { Ado } & \text { Adenosine } \\ \text { CI } & \text { Configurational interaction } \\ \text { DTT } & \text { Dithiotreitol } \\ \text { EDTA } & \text { Ethylenediaminetetraacetic acid } \\ \text { ESP charges } & \text { Electrostatic potential charges } \\ \text { ETDM } & \text { Electronic transition dipole moments } \\ \text { ETP } & \text { Energy transfer probabilities }\end{array}$




\begin{tabular}{|c|c|}
\hline FA & $\begin{array}{l}\text { Formycin A, 3-( } \beta \text {-D-ribofuranosyl) } \\
-7 \text {-aminopyrazolo }[4,3-\mathrm{d}] \text { pyrimidine }\end{array}$ \\
\hline FB & Formycin B \\
\hline FPLC & Fast protein liquid chromatography \\
\hline FRET & $\begin{array}{l}\text { Fluorescence resonance energy transfer } \\
\text { or Förester resonance energy transfer }\end{array}$ \\
\hline Guo & Guanosine \\
\hline Hepes & $\begin{array}{l}N \text {-2-hydroxyethylpiperazine-N'-2- } \\
\text { ethanesulfonic acid }\end{array}$ \\
\hline Ino & Inosine \\
\hline $\mathrm{m}^{7} \mathrm{Guo}$ & N (7)-methylguanosine \\
\hline MD & Molecular dynamics \\
\hline MM & Molecular mechanics \\
\hline $\mathrm{P}_{\mathrm{i}}$ & Orthophosphate \\
\hline PMSF & Phenylmethanesulfonyl fluoride \\
\hline PNP & Purine nucleoside phosphorylase \\
\hline PNP & $\begin{array}{l}\text { Escherichia coli hexameric PNP, } \\
\text { the product of the } d e o D \text { gene }\end{array}$ \\
\hline SA & Ammonium sulfate \\
\hline $\mathrm{SCF}$ & Self consistent field \\
\hline SDS-PAGE & $\begin{array}{l}\text { Sodium dodecyl sulfate polyacry } \\
\text { lamide gel electrophoresis }\end{array}$ \\
\hline TCSPC & Time-correlated single-photon counting \\
\hline Tris-HCl & $\begin{array}{l}\text { Tris (hydroxymethyl) aminomethane } \\
\text { hydrochloride }\end{array}$ \\
\hline 124 & Split valence, triple zeta basis set \\
\hline 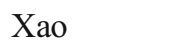 & Xanthosine \\
\hline
\end{tabular}

\section{Introduction}

$>$ Nonradiative Förster resonance energy transfer occurs from a donor (D) molecule to an acceptor (A) molecule, $\mathrm{D}^{*}+\mathrm{A} \rightarrow \mathrm{D}+$ $\mathrm{A}^{*}$, and results from a dipole-dipole interaction between their electronic states. Transfer occurs when the oscillations of an optically induced electronic coherence on $\mathrm{D}^{*}$ are resonant with the energy gap of A. FRET is a phenomenon used in molecular biophysical studies, including protein conformational changes, protein-protein interactions or protein-DNA interactions, as well as in fluorescence microscopy applications. Predicting FRET pathways in proteins using computer aided simulation techniques is very important for the reliable interpretation of experimental data. In this study, a novel and relatively simple methodology has been developed and applied to purine nucleoside phosphorylase (PNP) from E. coli complexed with a fluorescent ligand - formycin A (FA). Purine nucleoside phosphorylases (PNPs, E.C. 2.4.2.1) use orthophosphate $\left(\mathrm{P}_{\mathrm{i}}\right)$ to cleave the $\mathrm{N}$-glycosidic bond of $\beta$-(deoxy) ribonucleosides to yield $\alpha$-(deoxy) ribose 1-phosphate and the free purine base. Escherichia coli PNP, the product of the deoD gene, which cleaves Ado more effectively than Ino and Guo, has been reported to migrate as a hexamer. This is in contrast to the trimeric PNPs, which accept xanthosine (Xao) with comparable efficiency to Guo and Ino, the usual physiological substrates for trimeric PNPs. Absorption and emission spectra of E. coli PNP result from the presence of tyrosine residues and are characterized by maxima around 277 and $305 \mathrm{~nm}$, respectively. Since FA exhibit absorption and emission spectra red-shifted relative to PNP, with the maxima at 295 and $340 \mathrm{~nm}$, respectively, one observes the existence of fluorescence resonance energy transfer (FRET) processes between an excited Tyr residue $\left(D^{*}\right)$ of PNP and FA (A) [1]. A ribbon model of $E$. coli PNP, with highlighted tyrosines and FA is presented in Fig. 1 (below):

Apart from the wild-type PNP containing Phe159 and Tyr160, three mutants have been cloned, extracted, purified, and studied with molecular spectroscopy methods. These are described in Table 1.

Whereas the wild-type PNP exhibit the FRET phenomenon, it is not observed for the above mentioned mutants. In particular, since FRET is not observed for mutant number 2, Tyr160 in its $\mathrm{S}_{1}$ excited state is identified as the most probable energy donor, and FA as the acceptor [1].

\section{Experimental materials and methods}

Formycin A and E. coli PNP wild type

Formycin A (FA), N-2-hydroxyethyl-piperazine-N'-2ethanesulfonic acid (Hepes), were products of Sigma Chemical Co. (St. Louis, MO, USA). All solutions were prepared with high-quality MilliQ water. Reagents were of the highest commercially available quality, and only those of spectral grade, checked by UV absorption and/or fluorescence emission, were employed.

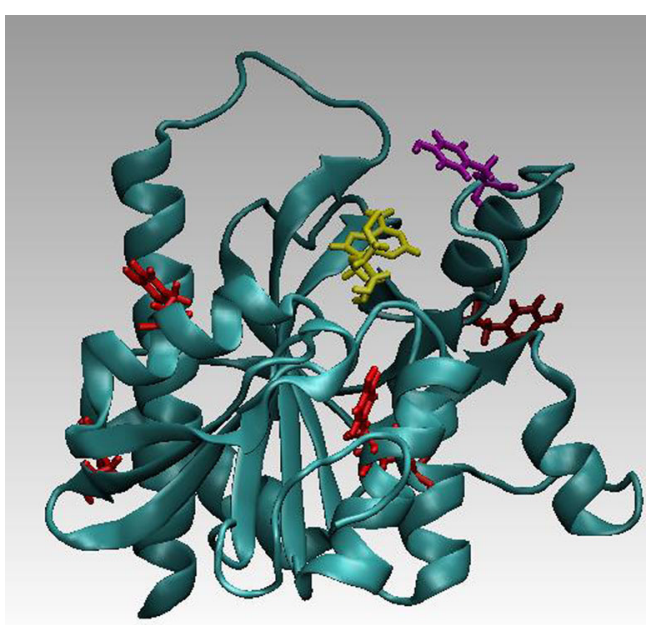

Fig. 1 E. coli PNP (ribbon representation) complexed with FA (yellow). The closest to FA tyrosine residue (Tyr160) is in magenta. Other tyrosines: Tyr27, Tyr52, Tyr72, Tyr186, and Tyr173 (going counterclockwise, when starting from the upper, left corner) are in red 
Table 1 E. coli purine nucleoside phosphorylase (PNP), the product of the $d e o D$ gene, and its mutants

\begin{tabular}{lll}
\hline Case & $\begin{array}{l}\text { Description of the } \\
\text { enzyme molecules }\end{array}$ & $\begin{array}{l}\text { Denotations with } \\
\text { one letter aminoacids } \\
\text { coding }\end{array}$ \\
\hline 1 & $\begin{array}{c}\text { Wild type PNP } \\
\text { (with Phe159 }- \text { Tyr160) }\end{array}$ & PNP \\
2 & $\begin{array}{l}\text { Tyr160 } \rightarrow \text { Ser160 } \\
\text { Phe159 } \rightarrow \text { Ala159 }\end{array}$ & PNP Y160S \\
3 & PNP F159A \\
4 & Phe159 $\rightarrow$ Tyr159 & PNP F159Y \\
\hline
\end{tabular}

The concentration of FA was determined spectrophotometrically at $\mathrm{pH} 7.0, \lambda_{\max } 294 \mathrm{~nm}\left(\varepsilon 10.3 \times 10^{3} \mathrm{M}^{-1} \mathrm{~cm}^{-1}\right)$. Measurements of $\mathrm{pH}(+/-0.05)$ were with a Jenway (UK) $\mathrm{pH}$-meter equipped with a combination semi-micro electrode and temperature sensor.

Cloned PNP from E. coli, the product of the deoD gene, was purified to apparent homogeneity and final specific activity about $100 \mathrm{U} \mathrm{mg}^{-1}$ [2]. The enzyme consists of six identical subunits, and enzyme concentrations are expressed in terms of the native hexamer $\left(\lambda_{\max } 277, \varepsilon 43.2 \times 10^{3} \mathrm{M}^{-1} \mathrm{~cm}^{-1}\right)$, unless otherwise stated. The molar extinction coefficient for the native hexamer $\varepsilon$ $43200 \mathrm{M}^{-1} \mathrm{~cm}^{-1}$ was determined [1] and a molecular mass of $159 \mathrm{kDa}$ calculated from the amino acid sequence [3]. Enzyme activity was monitored at $25^{\circ} \mathrm{C}$ in $50 \mathrm{mM}$ phosphate buffer (pH 7.0), spectrophotometrically by the coupled xanthine oxidase procedure with Ino as a substrate [4,5], and/or by following the changes in absorption of $\mathrm{m}^{7}$ Guo at $260 \mathrm{~nm}$ as the substrate [6].

\section{PNP mutants}

\section{Plasmid constructs}

The E. coli strain with pSE380 plasmid carrying the E. coli PNP DeoD gene was a kind gift from Dr. Joanne L. Turnbull. The plasmid was a template in site-directed mutagenesis reaction performed with the use of Invitrogen GeneTailor ${ }^{\mathrm{TM}}$ SiteDirected Mutagenesis System (Invitrogen) according to the manufacturer's protocol. Forward primers used for mutagenesis were designed as follows (changed TTC phenyloalanine codon underlined): 5'-ACCTGTTCTCCGCTGACCTGTA CTACTCTCCGG-3' (for F159Y mutation) and 5'-TGTTCT CCGCTGACCTGGCCTACTCTCCGG-3' (for F159A mutation). The reverse primer was the same for both reactions: 5'-CAGGTCAGCGGAGAACAGGTTACCCACGCG-3'. The synthesis of oligonucleotide primers and sequencing of the mutated PNP gene were done by Genomed S.A.

\section{Overexpression of mutated PNP}

E. coli $\mathrm{DH} 5 \alpha^{\mathrm{TM}}-\mathrm{T} 1{ }^{\circledR}$ cells containing pSE380 plasmid with the PNP F159Y or PNPF159A gene were grown in a
Superbroth medium $(2 \%$ peptone, $0.2 \%$ sodium phosphate (dibasic), $0.1 \%$ potasium phosphate (monobasic), $0.8 \%$ sodium chloride, $1.5 \%$ yeast extract, $0.2 \%$ glucose, and $50 \mu \mathrm{g} / \mathrm{ml}$ ampicillin) at $37{ }^{\circ} \mathrm{C}$ with constant shaking $(250 \mathrm{rpm})$ to $\mathrm{OD}_{600}$ of 0.8 before inducing the protein overproduction with $1 \mathrm{mM}$ isopropyl $\alpha$-D-1-thiogalactopyranoside (IPTG). The cells were grown for an additional $4 \mathrm{~h}$ in the same conditions and harvested by centrifugation for $20 \mathrm{~min}$ at $4000 \times \mathrm{g}$ at $4{ }^{\circ} \mathrm{C}$.

\section{Purification of F159Y}

The cell pellet, obtained from 61 of bacterial culture, was resuspended in $100 \mathrm{ml}$ of homogenizing buffer $\mathrm{H}(100 \mathrm{mM}$ Tris-HCl, pH 7.6, 2 mM EDTA, $10 \%$ glycerol (v/v), 1 mM DTT, $50 \mathrm{mM} \mathrm{NaCl}, 0.4 \mathrm{mg} / \mathrm{ml}$ lysozyme, and $0.015 \mathrm{mg} / \mathrm{ml}$ PMSF), incubated on ice for $30 \mathrm{~min}$, sonicated and centrifuged for $20 \mathrm{~min}$ at $43,000 \times \mathrm{g}$ at $4{ }^{\circ} \mathrm{C}$. The supernatant was filtered through four layers of gauze, supplemented with an equal volume of $100 \mathrm{mM}$ Tris-HCl buffer ( $\mathrm{pH}$ 7.6) containing 2 mM EDTA, $10 \%$ glycerol (v/v), and 1 mM DTT (buffer A) and applied onto Q-Sepharose ${ }^{\mathrm{TM}}$ Fast Flow column $(120 \mathrm{ml}$, GE Healthcare). The column was washed with 1.21 of buffer A and the enzyme was eluted with a 1.21 linear gradient of $0.05-0.3 \mathrm{M} \mathrm{KCl}$ in buffer A. Fractions were assessed for PNP activity (spectrophotometric assay) and purity (SDS-PAGE). The purest fractions were pooled $(235 \mathrm{ml})$, concentrated in Amicon stirred cell (Millipore) to $56 \mathrm{ml}$ and supplemented with an equal volume of $2 \mathrm{M}$ ammonium sulfate (SA). The enzyme was applied onto Phenyl-Sepharose CL-4B (30 ml, GE Healthcare) equilibrated with $50 \mathrm{mM}$ Hepes $\mathrm{pH} 7.6$ containing 1 M SA. The column was washed with 11 of the same buffer and PNP was eluted with a 11 linear gradient of 1-0 M $\mathrm{SA}$ in $50 \mathrm{mM}$ Hepes $\mathrm{pH}$ 7.6. The purest fractions were pooled (224 ml), concentrated in Amicon stirred cell to $14 \mathrm{ml}$, and dialyzed against $10 \mathrm{mM}$ Hepes $\mathrm{pH} 7.0$ with two changes of buffer. The enzyme was applied onto DEAE-cellulose $(20 \mathrm{ml}$, Whatman) washed with $250 \mathrm{ml}$ of $50 \mathrm{mM}$ Hepes $\mathrm{pH} 7.0$ and then, with $100 \mathrm{ml}$ of $100 \mathrm{mM}$ Hepes pH 7.0. PNP F159Y, the protein was eluted with $800 \mathrm{ml}$ linear gradient of $0-0.3 \mathrm{M}$ $\mathrm{NaCl}$ in buffer $\mathrm{A}$. The fractions containing PNP protein were pooled $(150 \mathrm{ml})$, concentrated in Amicon stirred cell and dialized against two changes of $50 \mathrm{mM}$ Hepes $\mathrm{pH}$ 7.0. The final PNP preparation $(7.5 \mathrm{ml})$ contained $32 \mathrm{mg}$ of protein per $\mathrm{ml}$ and was over $98 \%$ homogenous. The protein was divided into small aliquots and kept frozen at $-20{ }^{\circ} \mathrm{C}$ until further use.

\section{Purification of F159A}

The enzyme was purified using FPLC ÄKTApurifier 10 system (GE Pharmacia). The 21 bacterial culture was divided into $500 \mathrm{ml}$ aliquots, centrifuged and pellets were frozen at $-20^{\circ} \mathrm{C}$. The pellet of cells corresponding to $500 \mathrm{ml}$ of bacterial culture 
was resuspended in $10 \mathrm{ml}$ of buffer $\mathrm{H}$, incubated on ice for $30 \mathrm{~min}$, sonicated and centrifuged for $20 \mathrm{~min}$ at $43,000 \times \mathrm{g}$ at $4{ }^{\circ} \mathrm{C}$. The supernatant was supplemented with an equal volume of buffer A passed through MF Millipore membrane mix cellulose esters hydrophilic $0.45 \mu \mathrm{m}$ filter and applied onto HiLoad 16/10 Q-Sepharose HP column (20 ml) equilibrated with buffer A. The column was washed with $60 \mathrm{ml}$ of buffer A followed by $60 \mathrm{ml}$ of buffer A containing $150 \mathrm{mM} \mathrm{KCl}$. The enzyme was eluted with a $280 \mathrm{ml}$ linear gradient of $150-250 \mathrm{mM} \mathrm{KCl}$ in buffer A. The purest fractions, assessed by SDS-PAGE, were pooled and precipitated with SA ( $0-80 \%$ saturation). The protein pellet obtained by centrifugation at $47000 \times \mathrm{g}$ for $20 \mathrm{~min}$ at $4{ }^{\circ} \mathrm{C}$ was frozen at $-20{ }^{\circ} \mathrm{C}$. The above procedure was performed for each batch of bacterial pellet. The resulting protein pellet, corresponding to 21 of bacterial culture, was divided into two portions and subjected to further purification, one portion at a time. The pellet was resuspended in $50 \mathrm{mM}$ Tris- $\mathrm{HCl} \mathrm{pH} 7.6$ containing 1 M SA (buffer B), filtered as described above and loaded onto HiPrep 16/10 Phenyl-Sepharose column (20 ml resin). The column was washed with $40 \mathrm{ml}$ of buffer B and eluted with a $320 \mathrm{ml}$ linear gradient of $1-0 \mathrm{M} \mathrm{SA}$ in buffer B. The purest fractions were pooled and precipitated with $0-80 \%$ saturation of SA. The protein pellet was resuspended in $1 \mathrm{ml}$ of $50 \mathrm{mM}$ Hepes $\mathrm{pH} 7.0$ and dialyzed against three changes of the same buffer $(11$ for $1 \mathrm{~h}$, 11 for $3 \mathrm{~h}$ and 21 overnight). The final $2.9 \mathrm{ml}$ of homogenous PNPF159A preparation contained $31.6 \mathrm{mg}$ of protein. The protein was divided into small aliquots and kept frozen at $-20{ }^{\circ} \mathrm{C}$ until further use.

Steady-state absorption and fluorescence measurements

Ultraviolet absorption was monitored with a Varian (Australia) Cary 50 recording instrument, fitted with a thermostaticallycontrolled cell compartment at $25{ }^{\circ} \mathrm{C}$, using 5-mm pathlength cuvettes. Steady-state fluorescence emission and excitation spectra were measured with a Spex (USA) FluoroMax spectrofluorimeter at $25^{\circ} \mathrm{C}$, set up in the single photon counting mode, with 4-nm spectral resolution for excitation and emission. Samples were prepared in 500-570 $\mu \mathrm{l}$ of $50 \mathrm{mM}$ Hepes (pH 7, $\mathrm{pH} 8.3$ ), phosphate buffer ( $\mathrm{pH} 7, \mathrm{pH} 8.3)$, and acetate buffer $(\mathrm{pH} 4, \mathrm{pH} 5$, $\mathrm{pH} 6)$ in $5 \times 5-\mathrm{mm}$ Suprasil cuvettes. Fluorescence emission $\left(\lambda_{\mathrm{ex}}\right.$ 280, 295, and $313 \mathrm{~nm})$ and excitation $\left(\lambda_{\mathrm{em}} 305,340\right.$, and $365 \mathrm{~nm})$ spectra of PNP, FA, and their mixtures were recorded. Background emission $(\sim 0.5 \%)$, was eliminated by subtracting the signal for the buffer.

\section{Theoretical methods and simulations}

Molecular mechanics (MM) and dynamics (MD) simulations

MD simulations were carried out in the canonical ensemble for the wild-type E. coli PNP, complexed with FA, as well as for its two mutants with the following mutations: Phe159-> Ala159 and Phe159->Tyr159, denoted PNPF159A and PNPF159Y, respectively. NAMD/VMD (http://www.ks.uiuc. edu/Research/namd/) simulation MM and MD environment was used. CHARMM force field was applied (http://www. charmm.org/). Simulations accounted also for $\mathrm{S}_{1}$ excited states of Tyr160* (in PNP), Tyr159* (in PNPF159Y), as well as FA* in PNP. The assumption was for only one excited residue in each case under the study. This, in particular, meant that either Tyr160 or Tyr159 were excited. In addition, the deprotonated form of Tyr $160\left(\operatorname{Tyr}_{160} 0^{(-)}\right)$was considered in its ground as well as excited states. All MD simulations were carried out in periodic boundary conditions, with a box of the size $49 \times 78 \times 84 \AA$, containing the enzyme molecule surrounded by about $10^{*} 10^{3}$ water molecules and with ionic strength of $0.05 \mathrm{mM}$ model salt. MD simulations were proceeded by careful energy minimization with the norm of the energy gradient below $10^{-2} \mathrm{kcal} \mathrm{mol}^{-1} \AA^{-1}$. The crystallographic structure of E. coli PNP in complex with FA from Protein Data Bank (PDB code: 1PR1) was used for the modeling studies.

\section{Quantum mechanical (QM) calculations}

QM computations for Tyr and FA in their ground $\mathrm{S}_{0}$ as well as in excited $\mathrm{S}_{1}$ states were carried out using the TURBOMOLE 6.1 package (http://www.turbomole.com/) at the SCF-CI level. TZVP split valence, triple zeta basis sets was used (http:// www.gaussian.com/g_tech/g_ur/m_basis_sets.htm). Deprotonated $\mathrm{Tyr}^{(-)}$in its ground and excited states was also considered. In particular, the ESP charges were computed for all residues/molecules. Changes in the interaction potentials in $\mathrm{S}_{1}$ in comparison to $\mathrm{S}_{0}$ were accounted for by modifying the atomic charges. In the ground state, the CHARMM charges were applied. In the excited state, the CHARMM charges were modified by the changes of the quantum ESP charges when coming from the ground to the excited state. In this way the original CHARMM parameterization was applied including relative changes in the electrostatic interactions generated by the quantum-mechanical ESP charges when coming from the ground to the excited state or from the neutral to the deprotonated forms.

\section{Virtual titration}

In order to define initial conditions for MM and MD simulations at the microscopic level, the presence or absence of all protons at dissociable groups has to be defined. Typically, one assumes standard $\mathrm{pK}$ values for all aminoacids, and their total charges are determined, further resulting in well-defined atomic charges. In the present study, we discovered that the FRET phenomenon is sensitive to proton ionization states of residues in PNP, in particular in the area of the FA binding site. 
Therefore, PNP and its mutants in complex with FA were virtually titrated for $\mathrm{pH}$ in the range of 4.0-11.0. The PoissonBoltzmann formalism along with a Monte-Carlo procedure was used to determine mean electrostatic energies of protons in their binding-sites. The above-mentioned formalisms and numerical methodologies are presented, in particular, in $[7,8]$.

\section{Simulations of FRET processes}

MD simulations for the protein molecule containing $\mathrm{D}^{*}$ (here Tyr160*) and complexed with A (here formycin A) were carried out. Interactions of $\mathrm{D}^{*}$ with its molecular environment are accounted for by including changes of the ESP charges in $\mathrm{S}_{1}$, compared to $\mathrm{S}_{0}$, and computed at the SCF-CI level. $W_{F}$, frequency of FRETs per ns, depends on the inverse six-power of the $\mathrm{D}^{*}$-A distance, $R_{d a}$.

$W_{F}(t)=\frac{3 k^{2}(t)}{2 \tau_{d}}\left(\frac{R_{F}}{R_{d a}(t)}\right)^{6}$

where $\tau_{d}=2.57 \mathrm{~ns}$ is the experimental mean life-time of the donor. Förster radius $R_{F}$ is given by the following formula,

$R_{F}=0.2108\left(n^{-4} Q_{D}\left\langle k^{2}\right\rangle J\right)^{\frac{1}{6}}$

See Förster radius for tyrosine and aromatic ligand for its estimate. $R_{F}$ in $[\AA] . Q_{D}$ is the fluorescence quantum yield of the donor in the absence of the acceptor, $\kappa$ is the dipole orientation factor, $n$ is the refractive index of the medium, $J$ is the spectral overlap integral:

$J=\int_{0}^{\infty} f_{D}(\lambda) \varepsilon_{A}(\lambda) \lambda^{4} d \lambda$

where $f_{D}$ is the normalized fluorescence spectrum of $\mathrm{D}, \varepsilon_{A}$ is the molar excitation coefficient of the $A, \lambda$ is the wavelength in $[\mathrm{nm}]$.

The orientation factor $0<\mathrm{k}^{2}<4$ between $\mathrm{D}^{*}$ and A is computed and included in the analysis,

$k=\vec{\mu}_{d} \vec{\mu}_{a}-3\left(\frac{\vec{R}_{d a}}{R_{d a}} \vec{\mu}_{d}\right)\left(\frac{\vec{R}_{d a}}{R_{d a}} \vec{\mu}_{a}\right)$

$\mu_{\mathrm{d}}$ and $\mu_{\mathrm{a}}$ are normalized electric transition moments of the donor and acceptor, respectively. In the course of MD they can change their orientations. $R_{d a}$ is vector directed from $\mathrm{D}^{*}$ to $\mathrm{A}$. A few definitions of this vector have been considered, however, because final results did not depend much on the applied definition, a conventional, "spectroscopic definition" was applied. $R_{d a}$ was attached to the geometric center of the Tyr ring and directed to the mid point of the bond linking the six and five membered rings of FA.

The above quantities are time-averaged over the MD trajectories resulting in their time-averaged mean values,

$\left\langle k^{2}\right\rangle=\frac{1}{T} \int_{0}^{T} k^{2}(t) d t$

$\left\langle W_{F}\right\rangle=\frac{1}{T} \int_{0}^{T} W_{F}(t) d t$

One assumes ergodic hypothesis which states that the timeaverages are equal to ensemble averages, measured experimentally. The quantum yield of the energy transfer transition is given by:

$E\left(R_{d a}(t), k^{2}(t)\right)=\frac{1}{1+\left(w_{F}(t) \tau_{d}\right)^{-1}}$

and the mean quantum yield of the energy transfer is:

$\langle E\rangle=\frac{1}{T} \int_{0}^{T} \frac{1}{1+\left(W_{F}(t) \tau_{d}\right)^{-1}} d t$

Other possible deactivation paths, including thermal deactivation to the molecular environment, in particular water solvent, were also considered.

Similar methodology, using a combination of MC and MD simulations, has been applied to a model molecular system polyproline with two dyes attached to its ends ([9]; [10]). However, according to our knowledge, there are no studies based on first principles FRET models applied to such complex systems like PNP and its mutants, including protonationdeprotonation phenomena in the excited state of $\mathrm{D}^{*}$ (see below).

\section{Results and discussion}

Fluorescence properties of $E$. coli PNP and ligand (inhibitor)

The maxima of the absorption and emission spectra of PNPF159Y are located at 278 and $308 \mathrm{~nm}$, respectively (Fig. 2a), typical for proteins containing tyrosine, but no tryptophan [11], consistent with the fact that each of the subunits of that PNP mutant contains seven tyrosine residues and no tryptophan [12]. However, the fluorescence maximum is slightly shifted to higher wavelengths compared to wild type PNP [13], accompanied by higher values of the half width of 


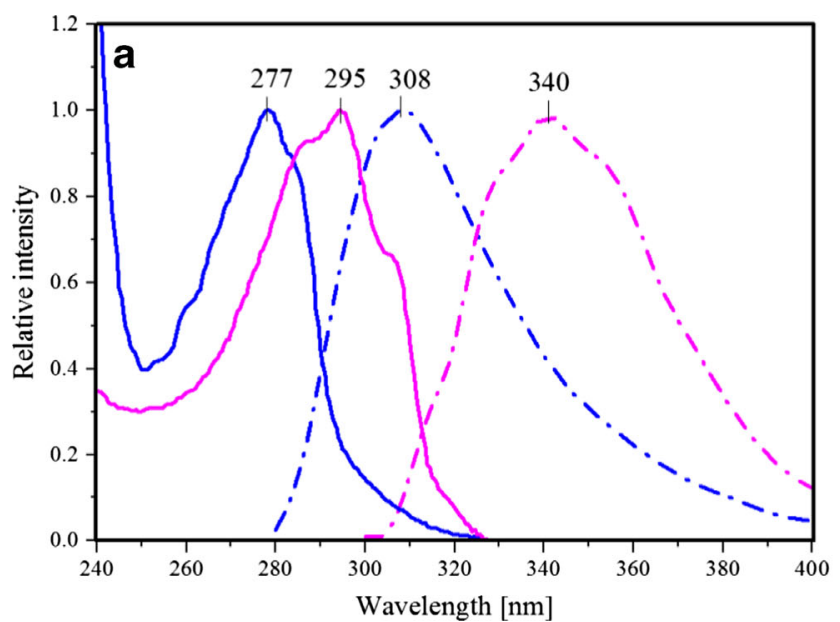

Fig. 2 UV absorption (solid) and fluorescence emission (dash-dot) spectra of E. coli PNP mutatnts PNPF159Y, PNPF159A, and FA (magenta) in 50mM Hepes, pH 7: a PNPF159Y (blue), and neutral

emission band and long-wavelength extended absorption spectrum of PNPF159Y (Figs. 2a and 3). A probable explanation of mentioned behavior might be the presence of the tyrosinate anion, which exhibits excitation maximum at $295 \mathrm{~nm}$ and emission shifted to long wavelengths (352 nm in solution) [14]. Indications for the presence of the tyrosinate anion in PNPF159Y are visualized by absorbance and emission spectra of the wild type and both mutants (Fig. 3), where aforementioned characteristic features are present. On the contrary, emission maximum of PNPF159A is at $306 \mathrm{~nm}$, which is close to the wild type PNP (Fig. 2b). By contrast, the nucleoside ligand FA, specific inhibitor of the bacterial enzyme $[15,16]$, exhibits absorption and emission spectra red-shifted relative to wt PNP and its mutants, with the maxima at 295 and $340 \mathrm{~nm}$, respectively (Fig. 2).

This permits selective excitation of FA in the enzyme-ligand complexes at excitation wavelengths above $295 \mathrm{~nm}$ (where tyrosine residues do not absorb). Selective observation of

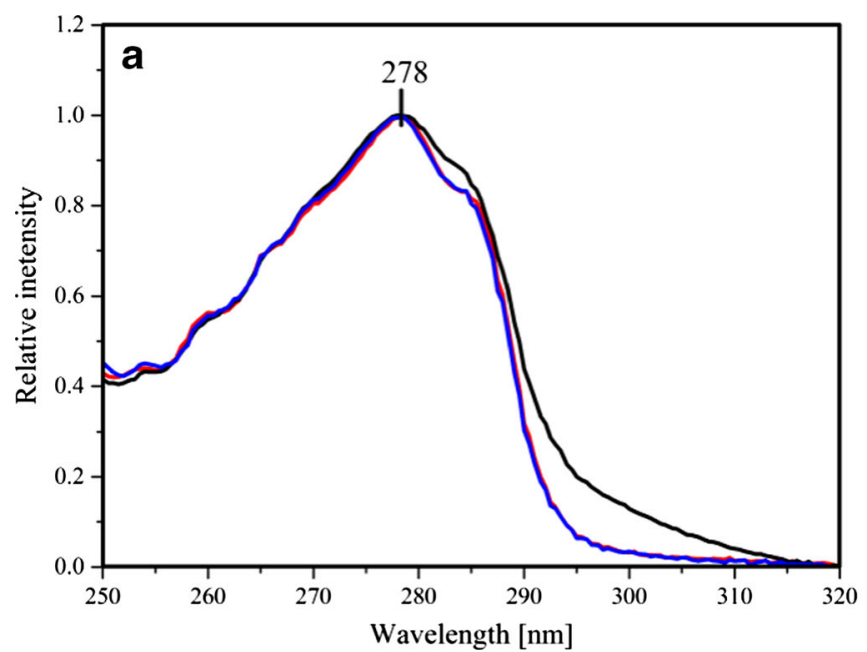

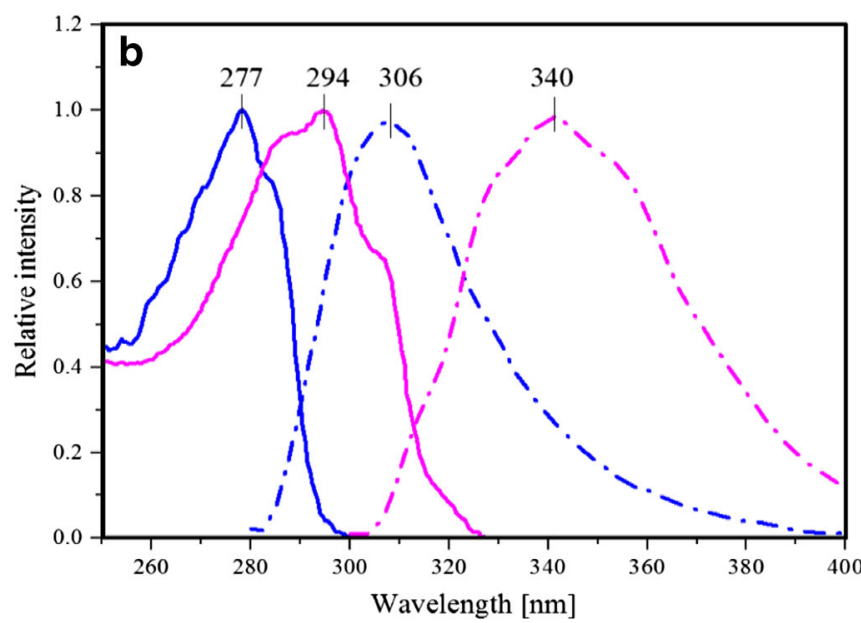

form of FA (magenta); b PNPF159A (blue), and neutral form of FA (magenta). Maximum intesities of the absorption and emission spectra of PNP $\left(\lambda_{\mathrm{ex}} 270 \mathrm{~nm}\right)$ and FA $\left(\lambda_{\mathrm{ex}} 295 \mathrm{~nm}\right)$ are normalized to unity

PNP fluorescence is possible in the range $290-310 \mathrm{~nm}$, and fluorescence of ligand (FA) at wavelengths above $360 \mathrm{~nm}$, where the contribution of protein fluorescence is usually negligible.

Förster radius for tyrosine and aromatic ligand

The existence of fluorescence resonance energy transfer (FRET) between protein tyrosine residues and a bound aromatic ligand should be consistent with the good alignment of the respective donor (tyrosine) emission and acceptor (FA) absorption spectral bands. The Förster radius was calculated as described elsewhere for other analogous systems [17], for FRET from Tyr residues to $\mathrm{m}^{1} \mathrm{FA}$ [1], which does not exhibit tautomerism. This gave $R_{F}=24 \AA$, comparable to values for similar systems, and to the dimensions of the enzyme subunit in the crystal [18]. Since the wt enzyme contains six tyrosine

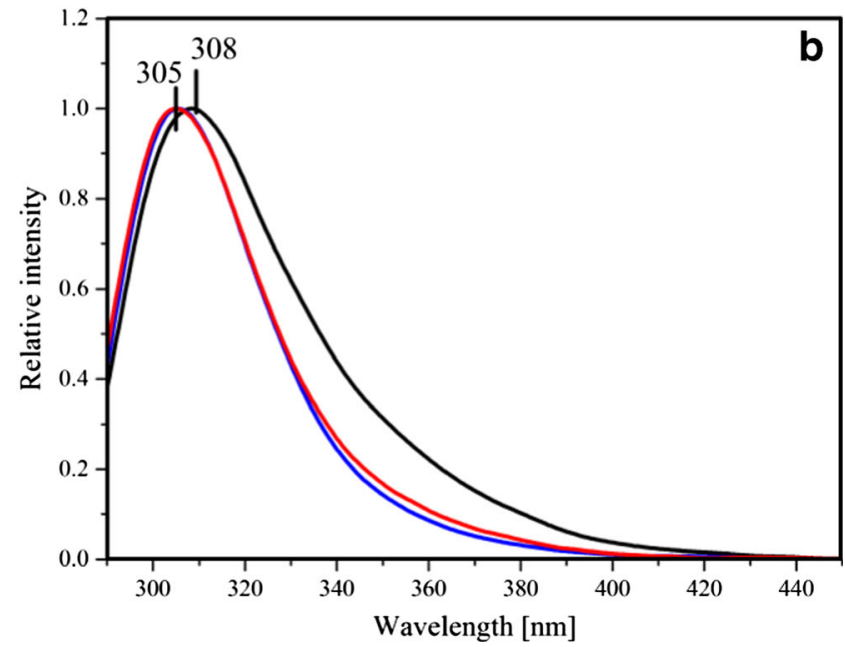

Fig. 3 Absorption a and emission after excitation at $280 \mathrm{~nm}$ b normalized spectra of wild type PNP (red), PNPF159Y (black), and PNPF159A (blue) 


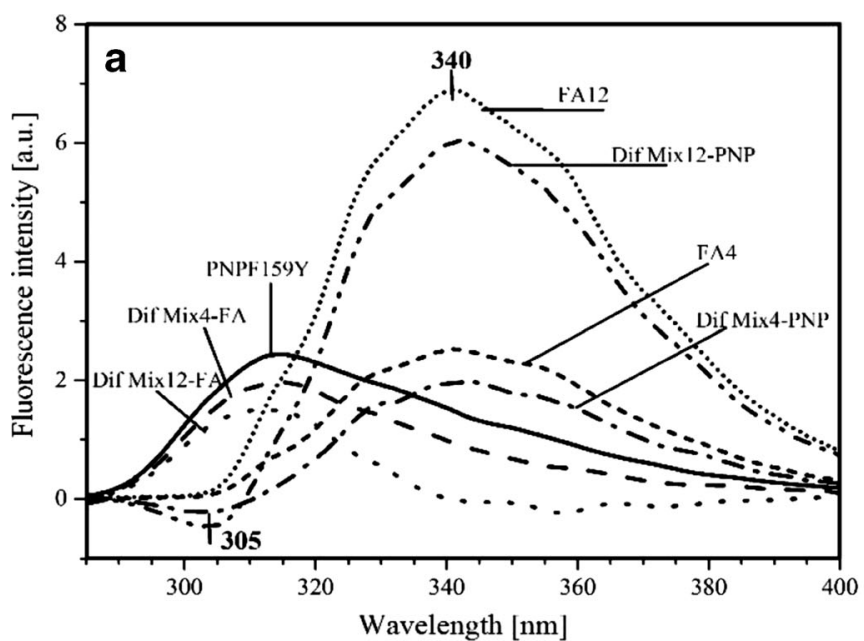

Fig. 4 Fluorescence emission ( $\lambda$ ex $280 \mathrm{~nm}$ ) spectra of PNPF159Y (a), PNPF159A (b), $4 \mu \mathrm{M}$ (FA4), and $12 \mu \mathrm{M}$ (FA12) FA in 50mM Hepes buffer ( $\mathrm{pH} 7)$, and fluorescence-emission difference $(\lambda \operatorname{ex} 280 \mathrm{~nm})$ spectra

residues per subunit, at least several of these should be located within range of the Förster distance to the acceptor.

\section{Absence of FRET between protein tyrosine residue(s) and the bound FA}

Fluorescence emission of both PNPF159Y and PNPF159A mutants overlaps with the absorption of FA ligand, in the extent convenient for FRET incident (Fig. 2a and b). For both mutants, the overlap integral is sufficient for energy transfer occurrence, which should result in enhanced FA emission in the complexes [19].

However, emission (Fig. 4) and excitation (Fig. 5) fluorescence spectra show that in both mutants, instead of FRET the protein fluorescence quenching by FA is observed, without

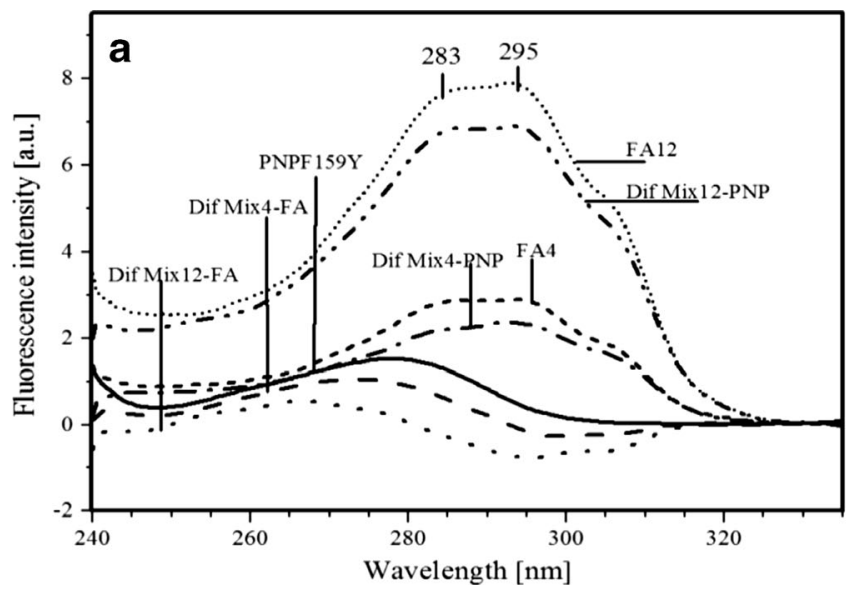

Fig. 5 Fluorescence excitation ( $\lambda \mathrm{em} 340 \mathrm{~nm}$ ) spectra of PNPF159Y (a), PNPF159A (b), $4 \mu \mathrm{M}$ (FA4), and $12 \mu \mathrm{M}$ (FA12) FA in 50mM Hepes buffer $(\mathrm{pH} 7)$, and fluorescence-excitation difference $(\lambda \mathrm{em} 340 \mathrm{~nm})$

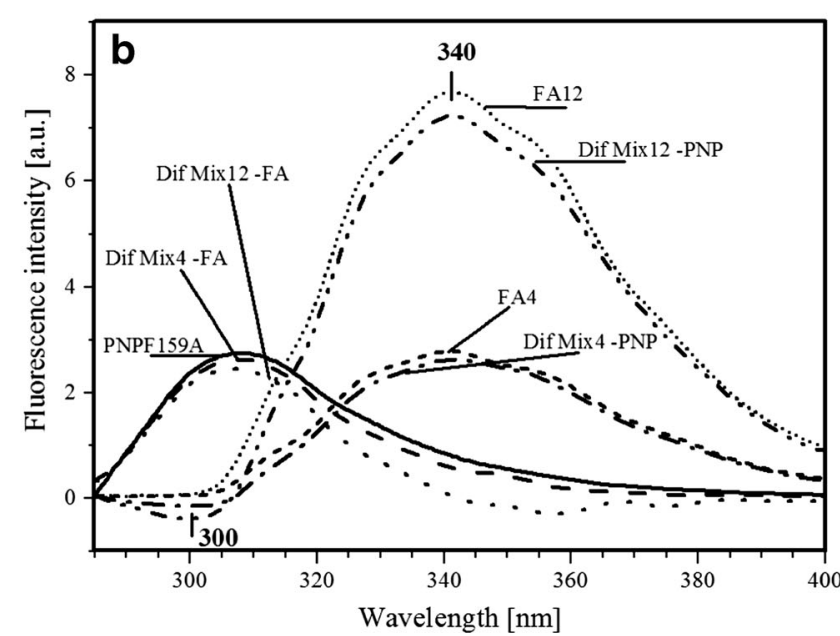

after subtraction of FA at both concentrations (Dif Mix4-FA, Dif Mix12FA), and after substraction of PNPF159Y (a), or PNPF159A b at both concentrations of FA (Dif Mix4-PNP, Dif Mix12-PNP)

affecting the location of the characteristic fluorescence maxi$\mathrm{ma}$ in proteins. This means that no changes in properties of aminoacid residues were caused by ligand binding (see difference-emission and difference-excitation spectra after FA subtraction).

However, as we can see in Figs. 4 and 5, the extent of enzyme quenching by FA depends on the type of mutant. The PNPF159Y fluorescence is much more sensitive for the FA presence, whereas PNF159A quenching is at least three times weaker than tyrosine mutant.

In addition, we can also observe quenching of ligands fluorescence (difference spectra after PNP substraction) that is caused by the protein mutants, and escalated with an increasing concentration of aforementioned, possibly due to extended interactions with the enzymes by creating complexes. As

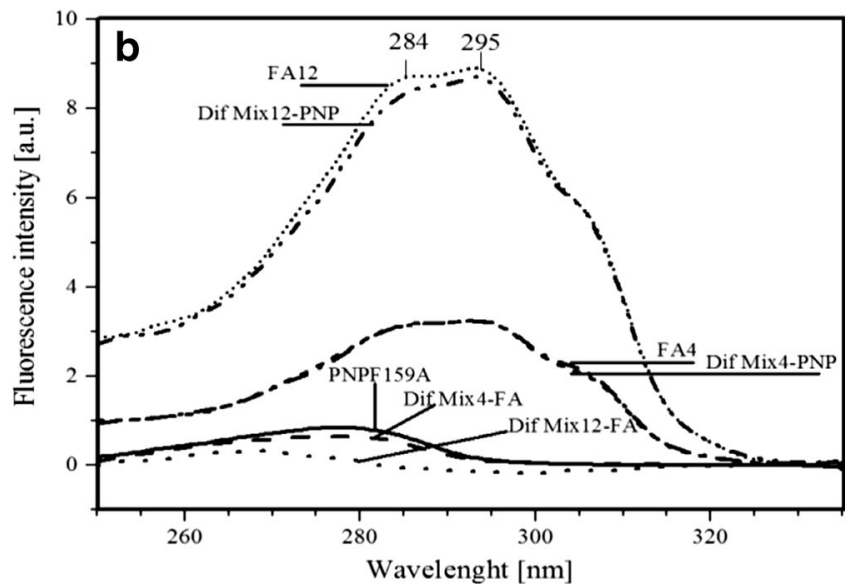

spectra after subtraction of FA at both concentrations (Dif Mix4-FA, Dif Mix12-FA), and after substraction of PNPF159Y (a), or PNPF159A b at both concentrations of FA (Dif Mix4-PNP, Dif Mix12-PNP) 

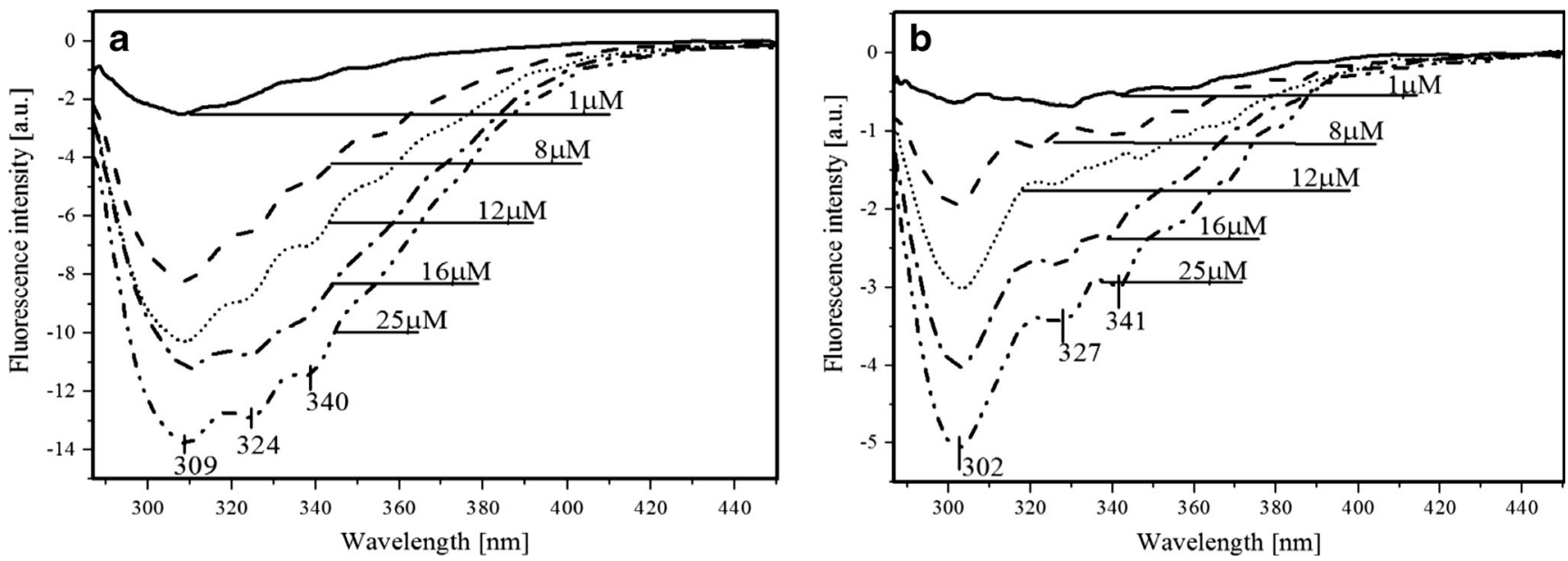

Fig. 6 Fluorescence-emission difference $\left(\lambda_{\mathrm{ex}} 280 \mathrm{~nm}\right)$ spectra after subtraction of different FA concentrations and PNPF159Y a or PNPF159A b in $50 \mathrm{mM}$ Hepes $\mathrm{pH} 7$

previously, this quenching depends on the type of mutant. Additionally, in Fig. 4 we observe blue-shift of the fluorescence minimum of both mutants. Probably, the reason is subtraction of ligands spectra, that are also quenched by interaction with the enzyme. This indicate the absence of the energy transfer between protein mutants and ligand, in contrast with considerations based on FRET in the wt PNP-FA complex, and supported by the difference fluorescence emission spectra of the mixture of wt PNP and FA. The latter clearly shows enhancement of FA fluorescence spectra due to the energy transfer from Tyr residues [1].

It is worth noting that the magnitude of quenching of FA fluorescence depends on the type of mutant. Both figures Figs. 4 and 5, demonstrates that decrease of FA emission is noticeably larger in the presence of PNPF159Y mutant than that of PNPF159A (Fig. 5a and Fig. B), respectively. The reason of aforementioned situation might be the presence of additional hydroxyl group in Tyr-159, which might change for example the transition moment or spatial arrangement of

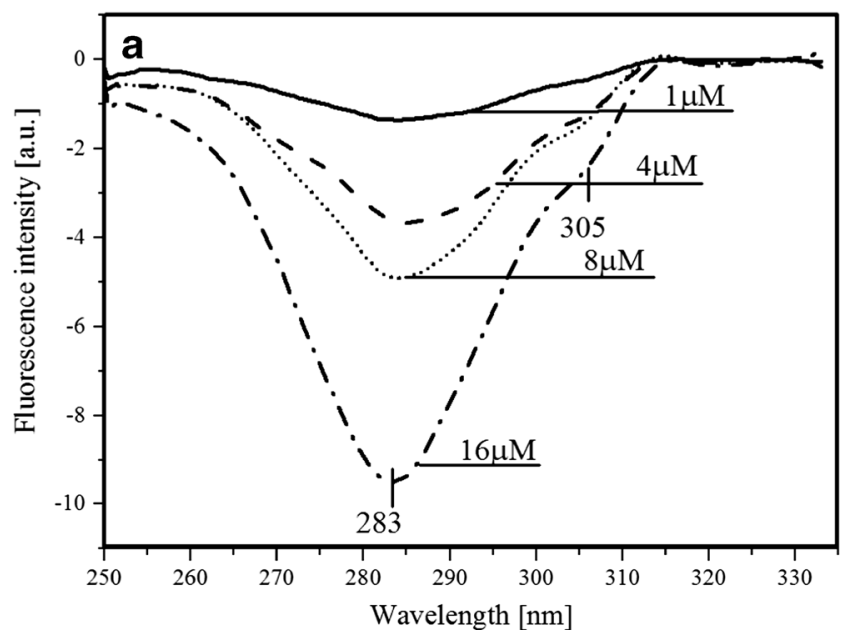

ligand and aminoacid residues in the active site, which could prevent energy transfer. In the case of PNPF159A mutant, the possible lack of energy transfer might be due to the absence of phenyl ring, the major difference between the wild type PNP and the PNPF159Y mutant. This could indicate an important role of the phenyl ring of Phe-159 in the energy transfer and in the binding of FA, which is supported by the high dissociation constant $\left(\mathrm{K}_{\mathrm{d}}\right)$ and thus the weak association of FA to PNPF159A. Additionally, we also observe concentrationdependent quenching of the FA fluorescence by both mutants, which is also in line with the absence of FRET.

Fluorescence-emission difference spectra after subtraction of both components (Fig. 6a and b) confirm quenching of the enzymes and ligand in both types of complexes that enhance with increasing ligand concentration. However, alanine mutant is much less susceptible for quenching by FA, probably due to the weaker binding of FA than in the tyrosine mutant. Moreover, the same situation is observed with FA, where quenching ratio between minimum of PNPF159Y/

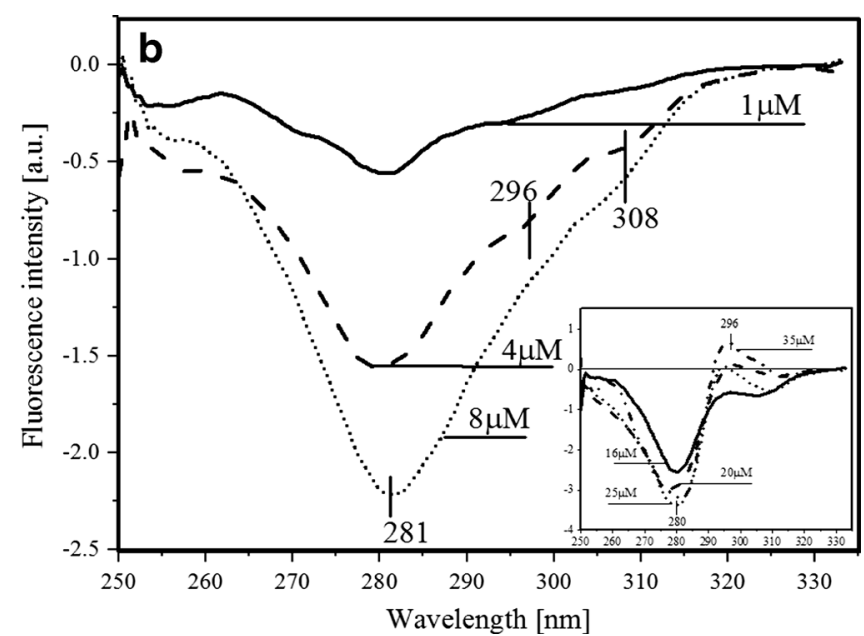

Fig. 7 Fluorescence-excitation difference $\left(\lambda_{\mathrm{em}} 340 \mathrm{~nm}\right)$ spectra after subtraction of different FA concentrations and PNPF159Y a or PNPF159A b in $50 \mathrm{mM}$ Hepes pH 7. 

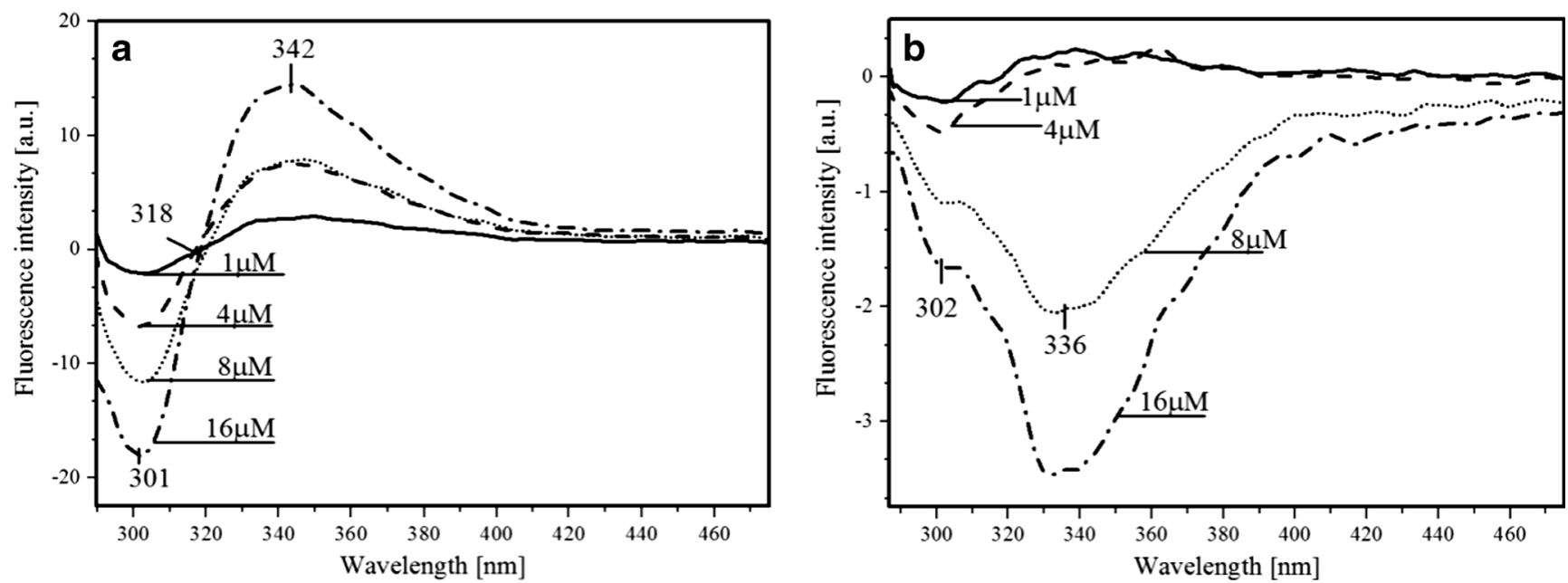

Fig. 8 Fluorescence emission difference $\left(\lambda_{\text {ex }} 280 \mathrm{~nm}\right)$ spectra of PNPF159Y a and PNPF159A b in 50mM acetate buffer, pH 5 after subtraction of the fluorescence emission spectra of FA in different concentrations and PNPF159Y a or PNPF159A b fluorescence emission spectra

FA and PNPF159A/FA differs considerably. It means that with tyrosine mutant not only FA associates better but due to the presence of additional hydroxyl group is firmly quenched.
Fig. 9 Structures, charges, and electric transition moments of tyrosine, its deprotonated form and formycin A. CHARMM charges in the ground state are in bold. Changes of the ESP charges after excitation from $S_{0}$ to $S_{1}$ are on the right site of the CHARMM charges. Arrows denote electric transition moments between $\mathrm{S}_{\mathrm{O}}$ and $\mathrm{S}_{1}$, computed using the SCFCI approximation with TURBOMOLE

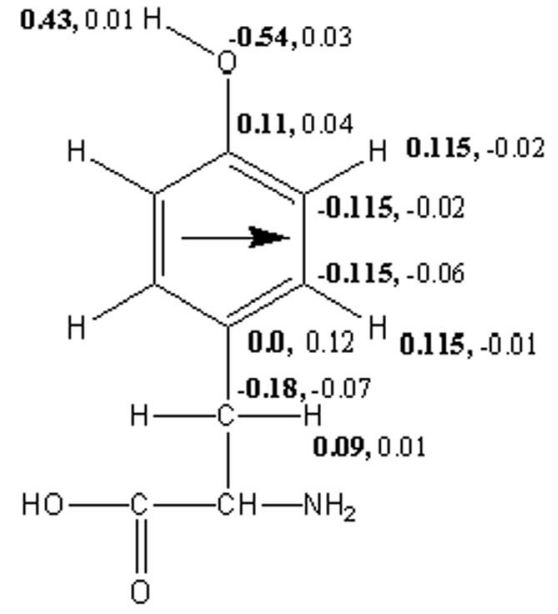

Tyrosine - neutral form<smiles>CCCCCCCCCCCCCCCCCCCCC(=O)O</smiles>

Tyrosine - deprotonated form

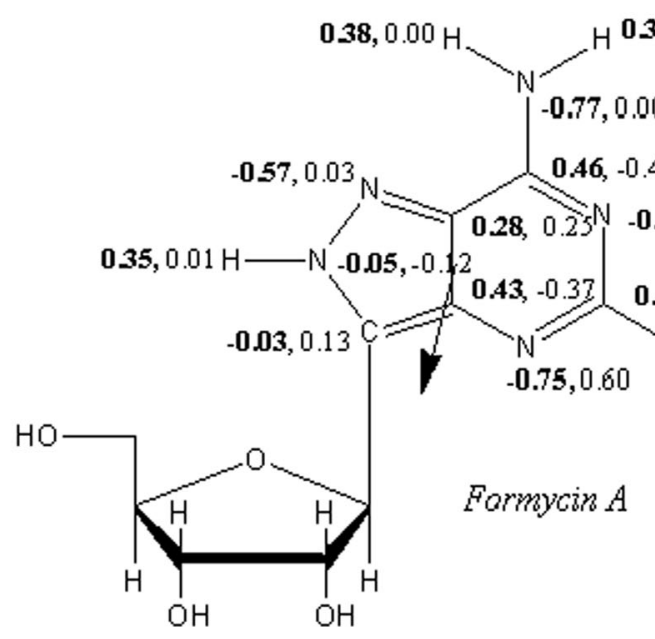


Table 2 FRET parameters calculated with the aid of QM computations and MD simulations, see Eqs. 1-8

\begin{tabular}{|c|c|c|c|c|c|}
\hline System & $<R_{d a}>$ & $<\kappa^{2}>$ & $\left(\kappa^{2}\right)_{\max }$ & $<W_{F}>$ & $<E>$ \\
\hline PNP, Tyr160* & $9.14 \pm 0.02$ & $0.558 \pm 0.004$ & 2.23 & $198.3 \pm 2.8$ & $0.938 \pm 0.002$ \\
\hline PNP F159A, Tyr160* & $6.50 \pm 0.02$ & $0.665 \pm 0.004$ & 1.81 & $2985.1 \pm 40.8$ & $0.985 \pm 0.001$ \\
\hline PNP F159Y, Tyr160* & $9.35 \pm 0.01$ & $0.809 \pm 0.005$ & 2.19 & $177.9 \pm 1.7$ & $0.977 \pm 0.001$ \\
\hline PNP, Tyr160 $60^{(-)} *$ & $9.24 \pm 0.01$ & $0.754 \pm 0.006$ & 3.36 & $\sim 0$ & $\sim 0$ \\
\hline PNP F159A, Tyr160 $0^{(-)} *$ & $9.39 \pm 0.01$ & $0.507 \pm 0.004$ & 1.95 & $\sim 0$ & $\sim 0$ \\
\hline PNP F159Y, Tyr160 ${ }^{(-)} *$ & $11.90 \pm 0.02$ & $1.165 \pm 0.006$ & 3.34 & $\sim 0$ & $\sim 0$ \\
\hline PNP F159Y $\mathrm{Y}^{(-)}, \operatorname{Tyr} 160^{(-) *}$ & $10.29 \pm 0.01$ & $0.820 \pm 0.003$ & 1.68 & $\sim 0$ & $\sim 0$ \\
\hline
\end{tabular}

$<R_{\mathrm{da}}>$ is the mean distance between the donor (Tyr160* or Tyr160 (-)*) and the acceptor (FA), in $[\AA] .<k^{2}>$ and $\left(k^{2}\right)$ max, are averaged and maximal orientation factors, respectively. $<W_{F}>$ is the mean frequency of FRETs per ns. $<E>$ is averaged quantum yield of the energy transfer. Standard error of the mean is also given. Zero values of $<E>$ result from the zero spectral overlap integral $J$ between $\operatorname{Tyr}^{(-)} *$ as $\mathrm{D}^{*}$ and $\mathrm{FA}$ as A, or alternatively from $R_{F}=0$ (Eqs. $1-2$ and 7-8)

Worth noting is the position of enzyme fluorescence minima. In Fig. 6a, it is $309 \mathrm{~nm}$, which proves that quenching concerns also the tyrosinate anion fluorescence. On the other hand, in alanine mutant minimum is located at $302 \mathrm{~nm}$, which indicate lack of the tyrosinate anion, and probable quenching of Tyr160 fluorescence.

Fluorescence-excitation difference spectra after subtraction of both components (Fig. 7a and b) also confirm quenching of the enzyme and ligand fluorescence. Nevertheless, slight shift of the excitation minima, from 277 to $281 \mathrm{~nm}$ (Fig. 7b) and $283 \mathrm{~nm}$ (Fig. 7a), may be attributed to the effect of nearby excitation band of FA fluorescence $\left(\lambda_{\mathrm{ex}} 295 \mathrm{~nm}\right)$. However, interesting observation is phenomenon with PNPF159A mutant with high FA concentrations (Fig. $7 b$ insert). We observe there a probable FRET, the quantity of which is quite small but noticeable. According to the previous assumptions, we can only see energy transfer at high concentrations of $\mathrm{FA}$, in line with its quite high $\mathrm{K}_{\mathrm{d}}$. value. It seems that only concentrations high enough to create numerous complexes can exert FRET, although quite small. The reason of low quantity of FRET is probably lack of phenyl ring at 159 position.

Smaller extent of FA quenching in the complex with the PNPF159A mutant than in the PNPF159Y mutant might indicate important role of the 159 phenyl ring in $\pi$-stacking interactions. This leads to the conclusion that FRET between Tyr160 and FA does not have to be direct but might occur via the phenyl ring of phenylalanine 159 in the wild type enzyme, see also [1]. On the other hand, the addition of the hydroxyl group might incorporate changes in the $\pi-\pi$ stacking, spatial arrangement of the aminoacid residues and ligand or might cause an orientation effect in the aspect of alignment between transition moments of mutant and FA. In addition, angle of $90^{\circ}$ can apparently cause total decay of energy transfer probability [19], and thus eliminating FRET when compared to the wild type PNP. On the contrary, the other possible scenario could be that the RET due quenching might be performed via polypeptide chain and ended by energy dissipation.

Moreover the lack of FRET and presence of the tyrosinate anion are explained by the low $\mathrm{pK}_{\mathrm{a}}$ value of tyrosine in $\mathrm{S}_{1}$ $4.2\left(\mathrm{pK}_{\mathrm{a}}\right.$ of the ground state tyrosine is 10.6), that is caused mostly by excitation and additionally by replacement of the Phe159 by the tyrosine residue. The latter destabilizes the active site environment and facilitates tyrosine deprotonation. Hence, in pH 7 tyrosine deprotonates to the tyrosinate anion, which has red-shifted emission spectrum where formycin almost does not absorb, and probably also causes enhanced thermal energy dissipation. This statement is supported by our studies of the mutants and wild type PNP in acetate buffer in $\mathrm{pH}$ 5, where FRET is observable in the PNPF169Y and not in the PNPF159A (Figs. 8a and b, respectively). The latter can be explained as the result of either lower affinity of this mutant to FA (high $\mathrm{K}_{\mathrm{d}}$ value) than in $\mathrm{pH} 7$, or the lack of FRET both probably caused by the absence of the phenyl ring of Phe159.

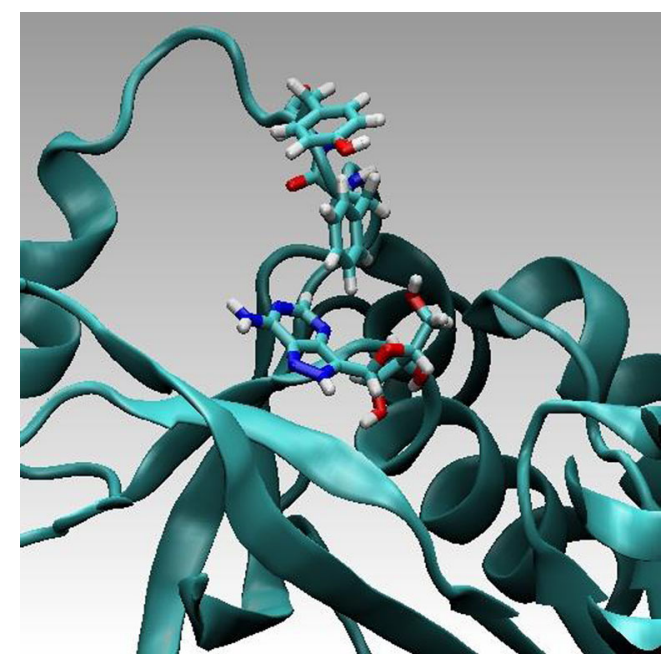

Fig. 10 E. coli PNP wild-type. Phe 159 stabilizes position of FA in the binding site. For such configurations FRET is very effective 
Fig. 11 The F159A mutant of E. coli PNP. The lack of the phenyl ring in the 159 position results in a weaker binding and higher mobility of the ligand. FA from time to time partially "flows-out" of the binding site which results in decreasing of FRET. Above are two characteristic configurations (left - optimal for FRET, right — not optimal one)
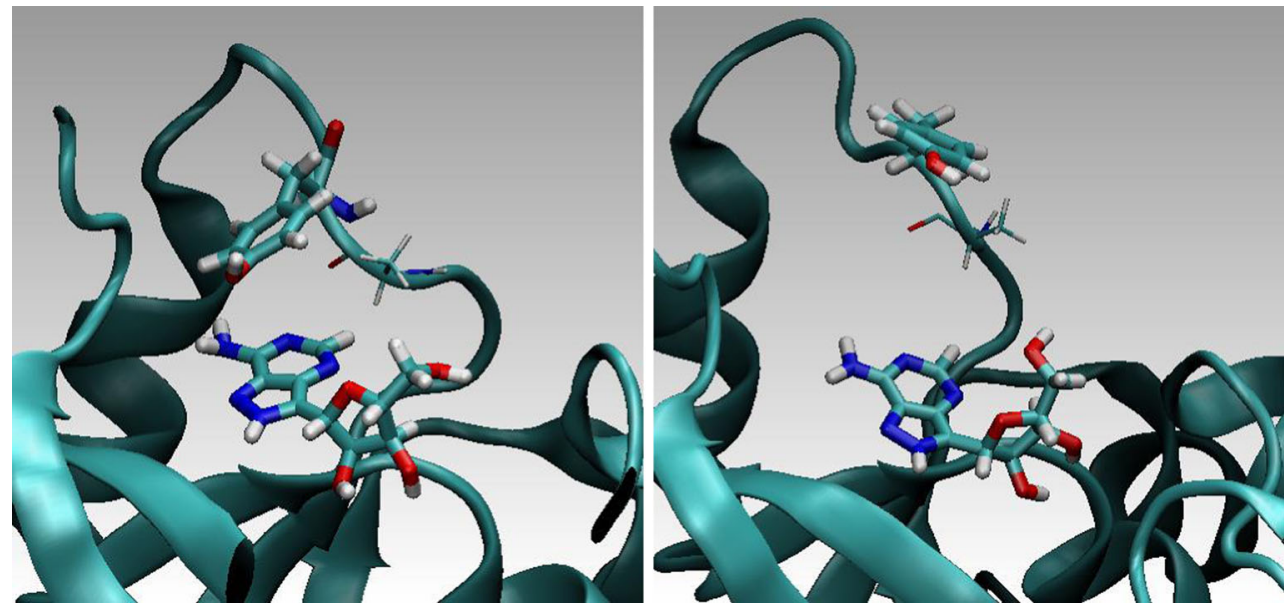

Quantum computations of atomic charges and electronic transition dipole moments

In order to carry out the molecular dynamics (MD) simulations, it was necessary to design parameters describing interactions between the enzyme molecule and FA in both the ground and excited states of FA and Tyr160. For this purpose, quantum-mechanical SCF-CI calculations of charge distributions in selected model systems mentioned in Methods have been carried out to estimate changes of atomic charges with respect to the reference charges present in the standard CHAR MM force fields, which were then modified by providing charges in excited states, and used in MD simulations.

The quantum-mechanical calculations involved the neutral molecules of Tyr, FA, and Ade, models of which have been generated using geometries from appropriate crystal structures. In particular, a model of the molecule of FA has been derived based on the crystal structure of the complex between the $E$. coli $\mathrm{PNP}$ enzyme, formycin $\mathrm{B}$, and the phosphate anion (PDB ID: 1PR1) [20]. The carbonyl oxygen on the $\mathrm{C} 6$ atom in the pyrimidine ring of formycin $\mathrm{B}$ has been replaced with the -
$\mathrm{NH}_{2}$ moiety, yielding formycin A. The geometry of the resultant molecule was initially optimized and then used in the QM calculations. Overall, the QM calculations allowed one to obtain ground-state point charges for all mentioned molecules and single-excitation-state point charges for Tyr and FA residues. The single-point calculations using the SCF-CI method and def-TZVP basis set [21] for all atoms have been carried out with $d s c f$ and egrad programs from the Turbomole 6.1 QM package [22]. The ESP partial charges [23] have been obtained by a fit to electrostatic potentials around the molecules. Additionally, electronic transition dipole moments (ETDM) for single-excitation transitions in Tyr and FA have also been computed with the same method. Atomic charges and ETDM are shown in Fig. 9 (below).

\section{Molecular dynamics simulations and FRET analysis}

Five ns MD simulations were carried out for complexes of the wild-type PNP and its above-mentioned mutants with FA. During this time-interval we did not observe any noticeable drift of the computed mean values (see standard error of the
Fig. 12 Wild-type PNP from E. coli in complex with FA. Characteristic are one of the most effective in FRET configurations (left) and an inhibiting one (right)

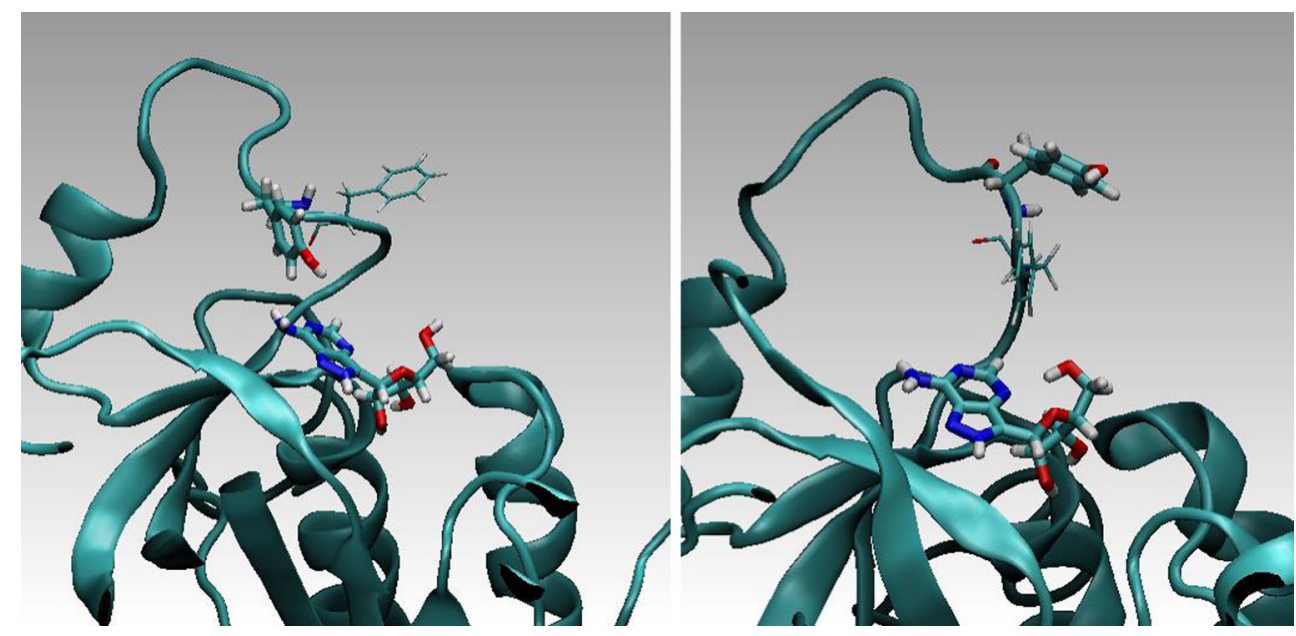


Fig. 13 Representative snapshots from the MD simulations for the PNPF159Y mutant with Tyr160 $(-) *$ and in complex with FA. Characteristics are one of the most effective in FRET configurations (left) and an inhibiting one (right)
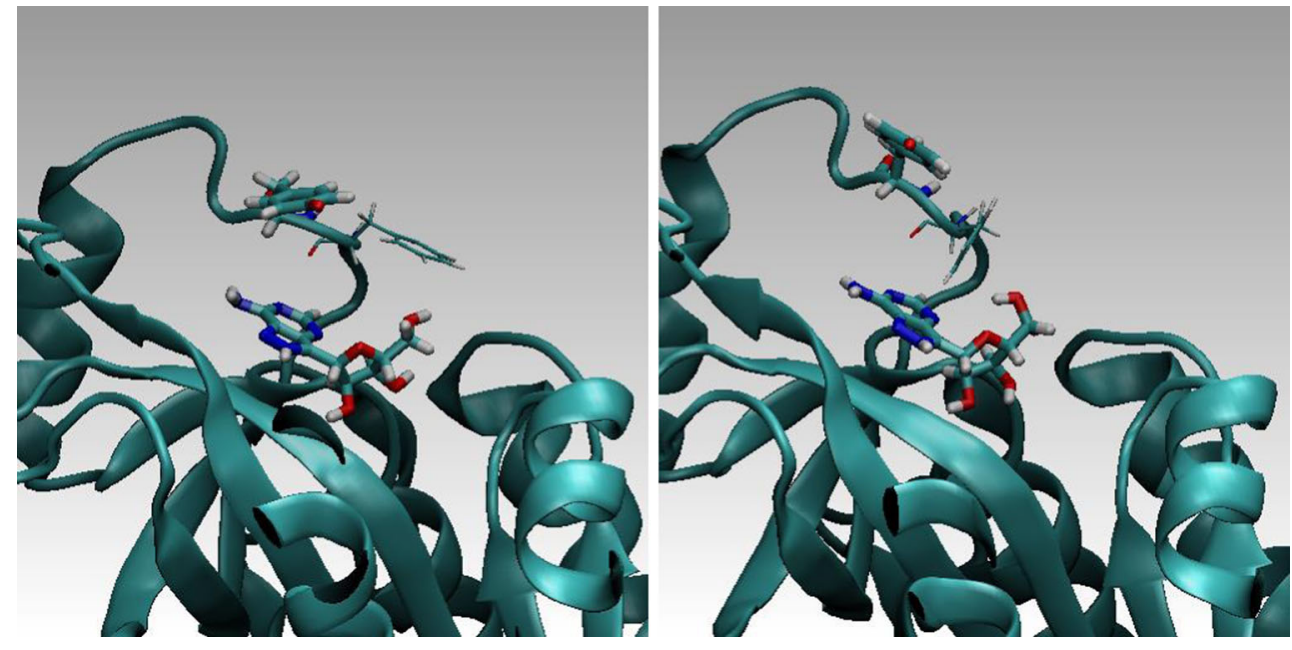

mean in Table 2). This indicates that the simulations were sufficiently long. MD simulations showed much better stability of the PNP (wild type) - FA complex in comparison to the PNP F159A mutant complex. In Figs. 10 and 11 (below) a few representative structural conformations in the area of the FA binding site are presented.

Based on MD simulations, energy transfer probabilities (ETP) were computed based on the non-radiative dipoledipole coupling model (Eqs. 1-5). This model results in the inverse six-power dependence of the donor-acceptor distance on ETP (Eq. 1-3). The orientation factor $\kappa^{2}$ of $\mathrm{D}$ and $\mathrm{A}$ was also computed and included in the analysis (Eq. 4). Total FRET probabilities were obtained by computing the FRET time-averages over the MD trajectories (Eqs. 5-8). Based on Eq. 1, the most effective and least effective configuration in FRET could be indicated and analyzed. Figure 12 (below) presents a characteristic effective and non-effective (inhibiting) configurations, left and right, respectively.

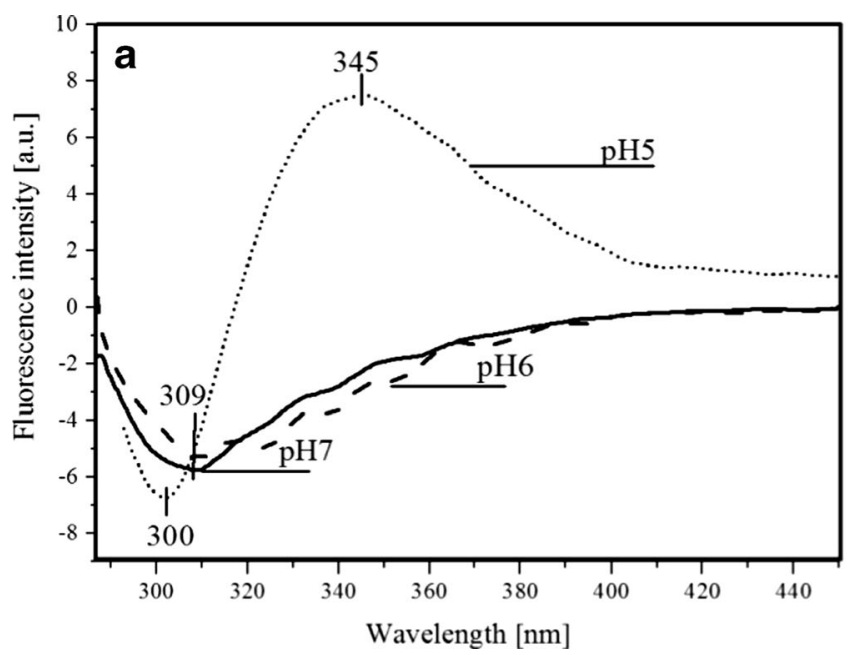

FRET efficiencies can be inferred from the photobleaching rates of the donor. Based on the above described formalism and MD simulations most important FRET parameters listed in "Theoretical methods and simulations" were calculated. They are collected in Table 2.

Obviously, the theoretical estimates for PNPF159A and PNPF159Y did not agree with experimental data. Based on the discussion above, we came to the conclusion that Tyr160* in these two mutants cannot exist in the neutral form. Deprotonation of this residue in its excited state explained the obvious discrepancies. The FRET parameters calculated for the following three cases, presented in Table 2, support this idea. In the case of Tyr $(-)^{*}$ the spectral overlap integral $J$ is close to zero (hence $R_{\mathrm{F}} \sim 0$ ) which makes the quantum yield of the energy transfer close to zero. Regardless of this, deprotonated Tyr160 ${ }^{(-)} *$ strongly interacts with the water environment leading most likely to the thermal deactivation of the tyrosinate anion. Figure 13 (below) shows a representative snapshot from

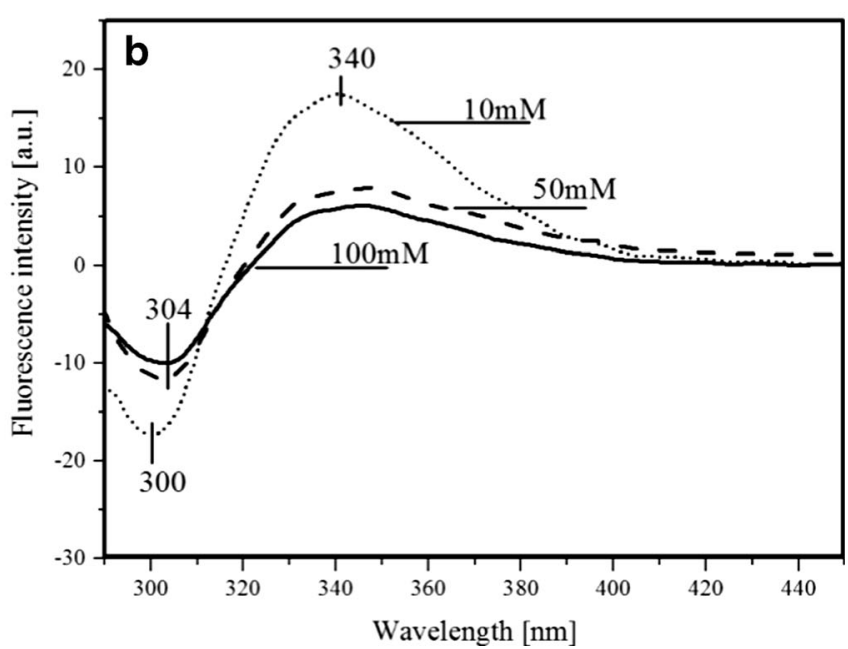

Fig. 14 Fluorescence-emission difference spectra of $3.9 \mu \mathrm{M}$ PNPF159Y mutant with $12 \mu \mathrm{M}$ FA, relative to the arithmetic sum of the two components: with a decreasing $\mathrm{pH}$ values from $\mathrm{pH} 7$ (Hepes buffer) to $\mathrm{pH} 6$ and $\mathrm{pH} 5$ (acetate buffer), and $\mathbf{b}$ decreasing concentrations of acetate buffer (pH 5) 
the MD simulations for the PNP F159Y mutant in the complex with FA which shows that Tyr160 (-)* is directed to the solvent and Tyr159 stabilizes location of FA.

\section{Conclusions}

The existence of FRET between Tyr160* and FA in the wild type PNP from E. coli and the lack of this phenomenon in the studied PNP mutants could not be easily explained. There were no obvious reasons why this phenomenon disappears in the mutants. In particular, when assuming existence of Tyr $160 *$ in its neutral state detailed theoretical simulations indicated existence of the FRET phenomenon. This lead us to the conclusion that in the mutants Tyr160* undergoes transition to $\operatorname{Tyr} 160^{(-)} *$, drastically changing the parameters defining FRET probabilities - compare results in Table 2. The tyrosinate anion exhibits fluorescence emission spectra redshifted $(c a .340 \mathrm{~nm})$ when compared to neutral tyrosine ( $c a$. $305 \mathrm{~nm}$ ). The lack of FRET is explained as the result of the low $\mathrm{pK}_{\mathrm{a}}(4,2)$ of the excited tyrosine in $\mathrm{S}_{1}$, hence at $\mathrm{pH} 7$ and above it is deprotonated, which also causes enhanced thermal energy dissipation. This statement is supported by our studies of the mutants and wild type PNP in an acetate buffer at $\mathrm{pH} 5$ where FRET is observable in PNPF169Y but not in PNPF159A (Fig. 8a and b). The latter can be explained as the result of either lower affinity of this mutant to FA (high $\mathrm{K}_{\mathrm{d}}$ value) or lack of FRET — both probably caused by the absence of the phenyl ring of Phe159. We have also observed that the quantum yield of FRET increases due to a decrease in $\mathrm{pH}$ from $\mathrm{pH} 7$ to $\mathrm{pH} 5$ (Fig. 14a) concomitantly with a higher population of the protonated tyrosine residues in their excited state. Additionally, the PNPF159Y fluorescence emission minimum is blue-shifted to $300 \mathrm{~nm}$ in pH 5 (Fig. 14a) which indicates both the presence of neutral tyrosine in position 159 and the positive effect of the FA emission band. On the other hand, an increasing concentration of the common proton acceptors like acetate or phosphate (present in the buffers) leads to weaker FRET (Fig. 14b), most probably due to an increasing concentration of the anionic form of tyrosine residues.

The red-shift of the tyrosinate anion emission and thus lack of, or very slight, spectral overlap integral and thermal energy dissipation are the reasons for the FRET absence in the studied mutants at $\mathrm{pH} 7$ and above. The presence of the tyrosinate anion results in a competitive energy dissipation channel and red-shifted emission, thus in consequence in the absence of FRET. These studies also indicate an important role of the phenyl ring of Phe159 for FRET in the wild-type PNP, which does not exist in the Ala159 mutant, and for the effective association of PNP with FA.

In a more general context, our observations point out very interesting and biologically important properties of the tyrosine residue in its excited state, which may undergo spontaneous deprotonation in the biomolecular systems, resulting further in unexpected physical and/or biological phenomena. Until now, this observation has not been widely discussed in the literature.

Acknowledgments These studies were supported by the Polish Ministry of Scientific Research and Higher Education (grant No. NN202105536). Simulations were carried out using computational infrastructure financed by the Biocentrum-Ochota project (POIG.02.03.00-000030/09) and by the Innovative Economy Grant (POIG.02.01.00-14-122/ 09). Quantum mechanical calculations were carried out in the Interdisciplinary Centre for Mathematical and Computational Modelling of the University of Warsaw.

Open Access This article is distributed under the terms of the Creative Commons Attribution License which permits any use, distribution, and reproduction in any medium, provided the original author(s) and the source are credited.

\section{References}

1. Kierdaszuk B, Modrak-Wójcik A, Wierzchowski J, Shugar D (2000) Formycin A and its N-methyl analogues, specific inhibitor of $E$. coli Purine nucleoside phosphorylase (PNP): induced tautomeric shift on binding to enzyme, and enzyme-ligand fluorescence resonance energy transfer. Biochim Biophys Acta 1476:109-128

2. Kierdaszuk B, Modrak-Wójcik A, Shugar D (1997) Binding of phosphate and sulfate anions by purine nucleoside phosphorylase from E. coli: ligand dependent quenching of enzyme intrinsic fluorescence. Biophys Chem 63:107-118

3. Hirshfield MS, Chaffe S, Koro-Johnson L, Mary A, Smith AA, Short SA (1991) Use of site-directed mutagenesis to enhance the epitopeshielding effect of covalent modification of proteins with polyethylene glycol. Proc Natl Acad Sci U S A 88:7185-7189

4. Kalckar HM (1947) The enzymatic synthesis of purine-ribosides. J Biol Chem 167:429-443

5. Stoeckler JD, Agarwal RP, Agarwal KC, Parks RE Jr (1978) Purine nucleoside phosphorylase from human erythrocytes. Methods Enzymol 51:530-538

6. Kulikowska E, Bzowska A, Wierzchowski J, Shugar D (1986) Properties of two unusual and fluorescent substrates of purine nucleoside phosphorylase: 7-methylguanosine and 7-methylinosine. Biochim Biophys Acta 874:355-363

7. Błachut-Okrasińska E, Lesyng B, Briggs JM, McCammon JA, Antosiewicz JM (1999) Poisson-Boltzmann model studies of molecular electrostatic properties of the cAMP-dependent protein kinase. Eur Biophys J 28:457-467

8. Antosiewicz J, Błachut-Okrasinska, E, Grycuk T., Lesyng B (2000) A correlation between protonation equilibria in biomolecular systems and their shapes: studies using a poisson-boltzmann model. In: Mathematical sciences and applications, GAKUTO International Series. Gakkotosho Co, Tokyo. 14:11-17

9. Hoefling M, Lima N, Haenni D, Seidel CAM, Schuler B, Grubmüller $\mathrm{H}$ (2011) Structural heterogeneity and quantitative FRET efficiency distributions of polyprolines through a hybrid atomistic simulation and Monte Carlo approach. PLoS ONE 6(5):e1979. doi:10.1371/ journal.pone.0019791

10. Hoefling M, Grubmüller H (2013) In silico FRET from simulated dye dynamics. Comput Phys Commun 184:841-852 
11. Ross JBA, Laws WR, Rousslang KW, Wyssbrod HR (1992) In: Lakowicz JR (ed.), Topics in fluorescence spectroscopy. Biochemical applications. Plenum, New York. 3:pp 1-63

12. Mao C, Cook WJ, Zhou M, Koszalka G, Krenitsky TA, Ealick SE (1997) The crystal structure of E. coli purine nucleoside phosphorylase: a comparison with human enzyme reveals a conserved topology. Sructure 5:1373-1383

13. Włodarczyk J, Stoychev Galitonov G, Kierdaszuk B (2004) Identification of the tautomeric form of formycin A in its complex with Escherichia coli purine nucleoside phosphorylase based on the effect of enzyme-ligand binding on fluorescence and phosphorescence. Eur Biophys J 33:377-385

14. Kierdaszuk B (2013) Fluorescence anisotropy of tyrosinate anion using one-, two- and three-photon excitation. J Fluoresc 23:339-347

15. Bzowska A, Kulikowska E, Shugar D (1990) Properties of purine nucleoside phosphorylase (PNP) of mammalian and bacterial origin. Z Naturforsch C 45:59-70

16. Bzowska A, Kulikowska E, Shugar D (1992) Formycins A and B and some analogues: selective inhibitors of bacterial (Escherichia coli) purine nucleoside phosphorylase. Biochim Biophys Acta 1120:239 247
17. Wu P, Brand L (1994) Resonance energy transfer: methods and applications. Anal Biochem 218:1-13

18. Koellner G, Luic M, Shugar D, Saenger W, Bzowska A (1998) Crystal structure of the ternary complex of $E$. coli purine nucleoside phosphorylase with formycin B, a structural analogue of the substrate inosine, and phosphate(sulphate) at 2.1 A resolution. J Mol Biol 280: $153-166$

19. Lakowicz JR (2006) Principles of fluorescence spectroscopy, 3rd edn. Springer, New York

20. Bennett EM, Li C, Allan PW, Parker WB, Ealick SE (2003) Structural basis for substrate specificity of Escherichia coli purine nucleoside phosphorylase. J Biol Chem 278:47110-47118

21. Eichkorn K, Weigend F, Treutler O, Alhrichs L (1997) Auxiliary basis sets for main row atoms and transition metals and their use to approximate Coulomb potentials. Theor Chem Accounts 97:119-124

22. Alhrichs R, Baer M, Haeser M, Horn H, Koelmel C (1989) Electronic structure calculations on workstation computers: the program system TURBOMOLE. Chem Phys Lett 162:165-169

23. Francl MM, Chirlian LE (2000) The pluses and minuses of mapping atomic charges to electrostatic potentials. Rev Comput Chem 14:131 